\title{
Novel MFSD8 Variants in a Chinese Family with Nonsyndromic Macular Dystrophy
}

\author{
Qin Xiang $\mathbb{D}^{1,2,3}$ Yanna Cao $\mathbb{D}^{4},{ }^{4}$ Hongbo Xu $\mathbb{D},{ }^{5}$ Zhijian Yang $\mathbb{D},{ }^{5}$ Liang Tang $\mathbb{D},,^{2,3}$ \\ Ju Xiang $\mathbb{D}^{2,3}$ Jianming $L i \mathbb{D},{ }^{1,6}$ Hao Deng $\mathbb{D}^{5},{ }^{5}$ and Lamei Yuan $\mathbb{D}^{5}$ \\ ${ }^{1}$ Department of Basic Biology, Changsha Medical University, Changsha, China \\ ${ }^{2}$ Center for Neuroscience and Behavior, Changsha Medical University, Changsha, China \\ ${ }^{3}$ Academics Working Station, Changsha Medical University, Changsha, China \\ ${ }^{4}$ Department of Ophthalmology, The Third Xiangya Hospital, Central South University, Changsha, China \\ ${ }^{5}$ Center for Experimental Medicine, The Third Xiangya Hospital, Central South University, Changsha, China \\ ${ }^{6}$ Department of Rehabilitation, Xiangya Boai Rehabilitation Hospital, Changsha, China \\ Correspondence should be addressed to Lamei Yuan; yuanlamei229@163.com
}

Received 30 November 2020; Revised 4 July 2021; Accepted 5 July 2021; Published 18 August 2021

Academic Editor: Alessandro Meduri

Copyright (c) 2021 Qin Xiang et al. This is an open access article distributed under the Creative Commons Attribution License, which permits unrestricted use, distribution, and reproduction in any medium, provided the original work is properly cited.

Purpose. To identify the molecular etiology of a Chinese family with nonsyndromic macular dystrophy. Methods. Ophthalmic examinations were performed, and genomic DNA was extracted from available family members. Whole exome sequencing of two members (the proband and her unaffected mother) and Sanger sequencing in available family members were performed to screen potential pathogenic variants. Results. Novel compound heterozygous variants, c.1066C $>\mathrm{T}$ (p.Pro356Ser) and c.1102+2T $>$ C, in the major facilitator superfamily domain containing 8 gene (MFSD8) were suspected to be involved in this family's macular dystrophy phenotype. The novel c.1066C $>$ T variant in the MFSD8 gene probably resulted in substitution of serine for proline at the 356th residue and was predicted to be "uncertain significance" through in silico analyses. The novel c. $1102+2 \mathrm{~T}>\mathrm{C}$ variant in the MFSD8 gene was likely to affect the splicing form and predicted to be "pathogenic." Conclusion. The novel compound heterozygous variants, c.1066C $>\mathrm{T}$ (p.Pro356Ser) and c.1102+2T>C, in the MFSD8 gene are likely responsible for the isolated macular dystrophy phenotype in this family. This study enlarged the MFSD8 gene mutant spectrum and might provide more accurate genetic counseling for this family.

\section{Introduction}

Inherited retinal dystrophies (IRDs) are comprised of a genetically heterogeneous group of ocular disorders with a wide clinical phenotypic spectrum, which have an approximated incidence of 1/3000-1/2000 [1,2]. Inherited macular dystrophies are clinical subtypes of IRDs, which are featured by bilateral central visual impairment, macula atrophy, and underlying retinal pigment epithelium degeneration $[3,4]$. Though the age of onset is variable, it often occurs in the first two decades of life [5]. Most of the diseases are rare and have common visual impairment and usually only show mild initial fundus alterations [3]. Autosomal dominant, autosomal recessive, $\mathrm{X}$-linked recessive, and mitochondrial inheritance patterns have all been described in macular dystrophies [5]. In more than 270 diseasecausing genes involved in IRDs, mutations in at least 32 genes including the major facilitator superfamily domain containing 8 gene (MFSD8, OMIM 611124) have been described to be associated with inherited macular dystrophies (the Retinal Information Network, RetNet, https://sph.uth. edu/Retnet/, last updated on January 21, 2021).

The MFSD8 gene, spanning approximately $48 \mathrm{~kb}$ and 13 exons on chromosome 4q28.2, encodes an MFSD8 protein with 518 amino acids [6]. The MFSD8 protein is a transmembrane lysosomal protein that possesses two N-glycosylation consensus sites, twelve membrane-spanning domains, and the cytosolic N- and C-terminus [7]. Recently, 
compound heterozygous MFSD8 variants, p.Glu336Gln/ c.1102G $>$ C and p.Glu336Gln/p.Glu381*, have been reported as being associated with nonsyndromic macular dystrophy [8].

Whole exome sequencing has recently been successfully applied to reveal the genetic causes of hereditary ocular diseases, especially disorders that are extremely genetically heterogeneous, such as IRDs [9, 10]. In this study, novel compound heterozygous variants, c.1066C $>\mathrm{T}$ (p.Pro356Ser) and c.1102+2T $>C$, in the MFSD8 gene were identified as the potential genetic cause of this Chinese family with nonsyndromic macular dystrophy by whole exome sequencing and Sanger sequencing.

\section{Subjects and Methods}

2.1. Subjects and Clinical Assessment. Four members of a nonconsanguineous Chinese family with one individual affected by macular dystrophy were recruited for this study (Figure 1(a)). Ophthalmic exams, including visual acuity measurement and fundus examination, were performed on the proband (II:1). After obtaining written informed consent, peripheral venous blood samples of four members (I:2, II:1, II:2, and II:3) were collected. Genomic DNA was extracted from lymphocytes using a FlexGen Blood DNA Kit (CWBIO, Beijing, China) according to the manufacturer's instructions. Ethical permission was allowed by the Institutional Review Board of the Third Xiangya Hospital, Central South University, Changsha, Hunan, China. As samples from human subjects were involved, this study was carried out adhering to the tenets of the Declaration of Helsinki.

2.2. Whole Exome Sequencing and In Silico Analyses. Genomic DNA samples of peripheral venous blood from the proband (II:1) and her unaffected mother (I:2) were used for whole exome sequencing. In brief, DNA fragments were separately obtained with sonication. Exons were captured using the xGen Exome Research Panel v1.0 (Integrated DNA Technologies, Inc., Coralville, IA, USA) following the manufacturer's instructions to establish exome libraries. Whole exome sequencing on the Illumina NovoSeq 6000 sequencing system and in silico analyses were completed in the Chigene (Beijing) Translational Medical Research Center Co., Ltd. (Beijing, China). The general process was as follows. Clean reads were mapped onto the human reference genome (GRCh37/hg19) through the Burrows-Wheeler Aligner (BWA, http://bio-bwa.sourceforge.net/). Variants were called by the Genome Analysis Toolkit (GATK, https:// software.broadinstitute.org/gatk/). SAMtools (http:// samtools.sourceforge.net) and Pindel (http://gmt.genome. wustl.edu/packages/pindel/) were subjected to call singlenucleotide polymorphisms and insertions/deletions, respectively. Detected variants were annotated with the ANNOVAR (ANNOtate VARiation) tool (http://www. openbioinformatics.org/annovar/). Variants were filtered referring to public databases including the Single Nucleotide Polymorphism database v147, the 1000 Genomes Project, the Exome Aggregation Consortium, and the National Heart, Lung, and Blood Institute Exome Sequencing Project 6500 with minor allele frequency no less than 5\%. The remained variants located in coding regions and canonical splice sites were taken for candidate identification. Several online programs including Protein Variation Effect Analyzer, Sorting Intolerant from Tolerant, Polymorphism Phenotyping v2, and MutationTaster were used to predict whether missense variants impacted protein functions $[11,12]$. The effects of splicing variants were predicted by Human Splicing Finder and MaxEntScan online programs $[13,14]$. Variants in genes associated with retinopathies were considered as potential disease-causing variants adhering to the standard variant interpretation guidelines of the American College of Medical Genetics and Genomics (ACMG) [15].

2.3. Sanger Sequencing. Variants screened by whole exome sequencing and suspected as potential causes were further validated in the proband and available family members. The polymerase chain reaction (PCR) amplification was performed with site-specific primers: $5^{\prime}$-TCCTGGTTATTTTTAGTGGAAAA- $3^{\prime}$ and $5^{\prime}$-TTGGAGACTTCCAAAGACCAA- ${ }^{\prime}$. Purified PCR products were directly sequenced using an $\mathrm{ABI}$ 3500 sequencer (Applied Biosystems, Foster City, CA, USA) adhering to manufacturer's instructions. The Chromas software (Technelysium Pty Ltd., Tewantin, QLD, Australia) was applied for analysis of sequence data.

2.4. Conservative Analysis. Multiple orthologous sequence alignments from seven different species were conducted by the online Clustal Omega tool (https://www.ebi.ac.uk/Tools/ $\mathrm{msa} /$ clustalo/) as described previously [16].

\section{Results}

3.1. Clinical Manifestations. The proband presented with a two-year history of bilateral progressive visual loss and was diagnosed with macular dystrophy at 18 years of age. She had an uncorrected visual acuity of 16/200 in her right eye and 20/200 in her left eye. Fundus photographs showed macular degeneration with discrete and large confluent macular drusen (Figure 2). She had normal mental and motor abilities and denied histories of seizures and myoclonus.

\subsection{Identification of MFSD8 Gene Variants in the Proband.} Whole exome sequencing and in silico analyses were performed on two members (the proband and her unaffected mother) of this family to seek the molecular etiology. Data with an average coverage of the target sequence no less than $20 \times$ accounted for $98.76 \%$ and $98.90 \%$, respectively. Mean sequencing depth on target for the two members was $120.46 \times$ and $101.43 \times$, respectively. A step-by-step bioinformatics pipeline containing base calling, low-quality read filtering, variant annotation, and biological function prediction was performed. In more than 270 known 


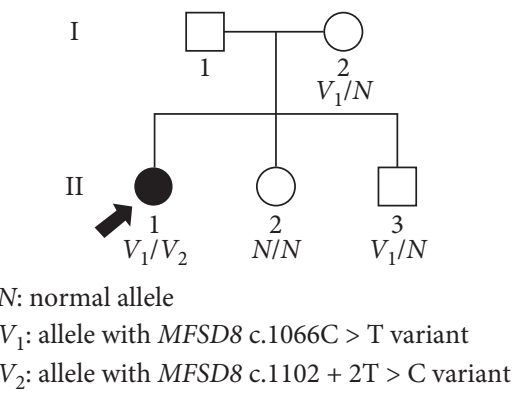

(a)

MFSD8 c. $1102+2 \mathrm{~T}>\mathrm{C}$

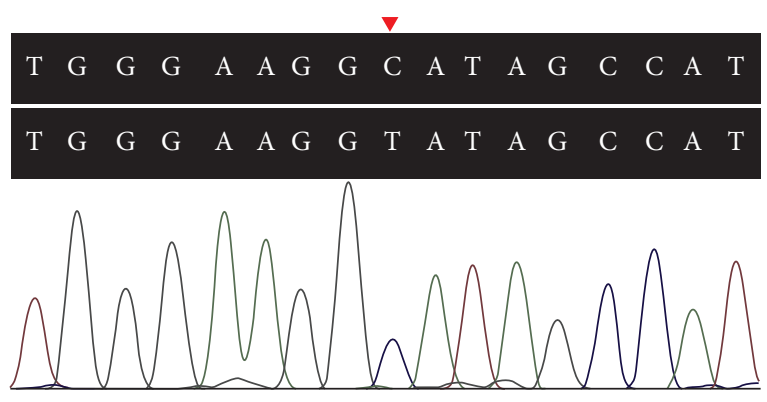

(c)

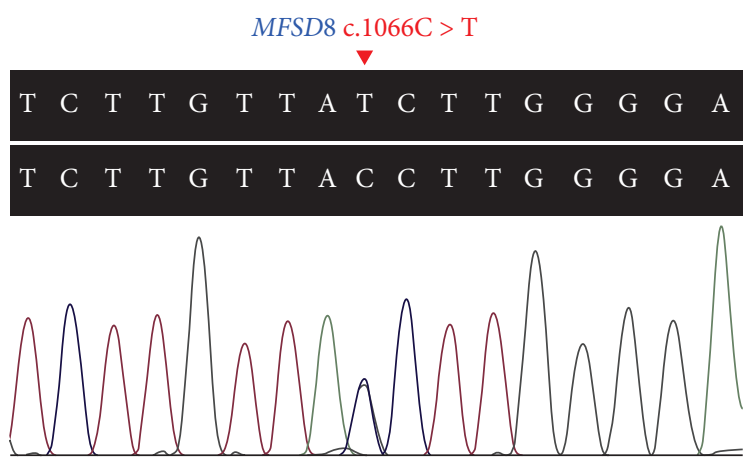

(b)

(d)

Figure 1: Compound heterozygous MFSD8 gene variants identified in a macular dystrophy family. (a) A Chinese macular dystrophy pedigree. Squares and circles represent males and females, respectively. The fully shaded circle represents the female patient. The arrow indicates the proband. (b) Sanger sequencing validated the heterozygous c.1066C $>$ T variant in the proband. (c) Sanger sequencing validated the heterozygous c.1102+2T $>\mathrm{C}$ variant in the proband. (d) Alignment of MFSD8 proteins in various organisms. The proline residue at position 356 of MFSD8 protein is highly conservative across seven different species. MFSD8, major facilitator superfamily domain containing 8 .

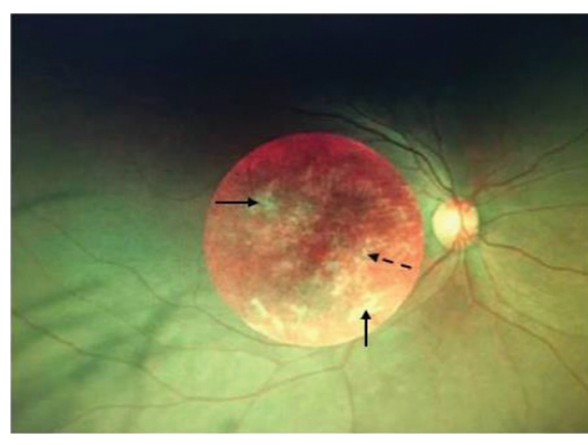

OD

(a)

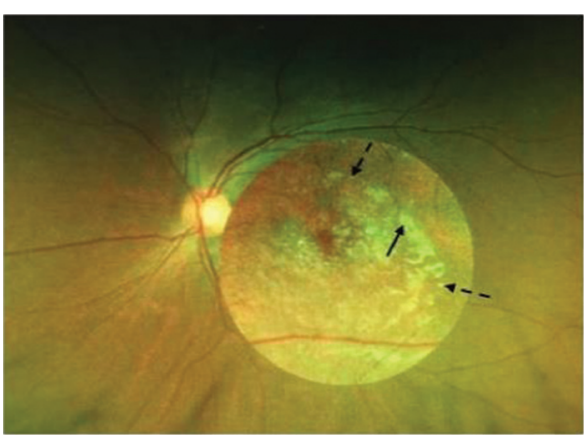

OS

(b)

FIGURE 2: Fundus photographs of the proband with macular dystrophy. Fundus photographs showed macular degeneration with discrete (dashed arrow) and large confluent (solid arrow) macular drusen. OD, right eye; OS, left eye.

retinopathies genes (based on RetNet), only two novel variants, c. $1066 \mathrm{C}>\mathrm{T}$ and $\mathrm{c} .1102+2 \mathrm{~T}>\mathrm{C}$, in the MFSD 8 gene (NG_008657.1 and NM_152778.4) were identified as being responsible for the macular dystrophy phenotype according to filtering steps of the methods. The compound heterozygous variants were further validated in the proband by Sanger sequencing (Figures 1(b) and 1(c)). The missense variant c.1066C $>\mathrm{T}$ (p.Pro356Ser) that was predicted by in silico analyses to probably damage the protein function was also detected in the proband's unaffected mother (I:2) and younger brother (II:3). Sequence alignment of multiple orthologous proteins revealed proline at position 356 of the MFSD8 protein was highly conservative in various organisms (Figure $1(\mathrm{~d})$ ). The splicing variant $\mathrm{c} .1102+2 \mathrm{~T}>\mathrm{C}$, located in the intron 11 of the MFSD 8 gene, was not detected by Sanger sequencing in available unaffected members. It was predicted to probably affect splicing due to the alteration of the donor site. According to the ACMG guidelines for 
sequence variants, c. $1066 \mathrm{C}>\mathrm{T}$ and c. $1102+2 \mathrm{~T}>\mathrm{C}$ variants were classified as "uncertain significance" and "pathogenic," respectively. All of the above indicated that the compound heterozygous variants were likely to be the genetic cause of the proband's macular dystrophy phenotype.

\section{Discussion}

This study identified novel compound heterozygous variants, c.1066C $>\mathrm{T}$ and c.1102+2T $>\mathrm{C}$, in the MFSD 8 gene as being possibly responsible for the family's macular dystrophy phenotype. In previous studies, the MFSD8 gene mutations were the most common genetic cause of variant late-infantile neuronal ceroid lipofuscinosis (vLINCL), also termed as ceroid lipofuscinosis neuronal 7 disease (CLN7, OMIM 610951), which had an onset age of 1.5 to 5 years [17]. Symptoms of vLINCL included visual loss, seizures, psychomotor retardation, and premature death [18]. Some patients with vLINCL displayed visual impairment early, indicating that the retina is more sensitive to the MFSD8 gene variant than the brain, and retinal neuron loss may precede brain neuron loss, supported by the findings in the $M f s d 8$ deficient mice [19]. Nevertheless, no hallmarks or severe neurologic symptoms were observed in the proband, supporting the MFSD8 gene as a nonsyndromic macular dystrophy gene [20]. The findings were further underpinned by several recent reports of compound heterozygous or homozygous MFSD8 gene variants identified in patients with nonsyndromic macular dystrophy, nonsyndromic retinitis pigmentosa, or more severe rod-cone dystrophy $[8,20,21]$.

The MFSD8 protein, a ubiquitous lysosomal membrane protein, is a member of the major facilitator superfamily transporter proteins that transport small solutes through transmembrane ion gradients [6]. However, MFSD8 protein substrates are not clearly understood. In the retina, the MFSD8 protein is located in photoreceptor synaptic terminals and may be involved in the formation of synaptic vesicles [21]. However, the precise molecular mechanisms are unclear. Mutations in the MFSD8 gene involved in a wide spectrum from hypomorphic to null alleles, and hypomorphic mutations could lead to partial function loss. According to the conjugation of these alleles, phenotypes are likely to vary from mild nonsyndromic retinopathies to severe syndromic retinopathies [1]. Intriguingly, the p.Glu336Gln variant, located proximately to p.Pro356Ser, was thought to be a 'mild' variant giving rise to isolated macular dystrophy when combined in trans with either a null variant or a missense variant [21]. The p.Met454Thr homozygous variant was thought to be a 'moderate' variant causing a more severe rod-cone dystrophy, but not vLINCL [21]. The p.Met454Thr variant, when paired with a more severe variant allele (p.Trp407Arg), may cause a severe neurological phenotype [22]. In the present study, the c.1066C $>$ T (p.Pro356Ser) variant was predicted to be of "uncertain significance," and it seemed to be a 'mild' variant. The c.1102+2T >C variant was predicted to be "pathogenic," and it probably affected splicing due to the alteration of the donor site. Similarly, a proximal c.1102G $>$ C variant in the MFSD8 gene was shown to result in a skipping of exon 11 and caused isolated macular dystrophy when paired with a c.1006G $>$ C (p.Glu336Gln) variant [8]. These findings are consistent with the previously proposed genotype-phenotype correlation that the two severe allelic variants result in vLINCL, whereas milder variants on at least one allele cause nonsyndromic retinal degeneration diseases [20].

Some pathogenic MFSD8 gene variants did not change the protein subcellular location in lysosomal compartments, suggesting that these variants probably altered protein stability or even functional property in lysosomes [7, 23]. The p.Pro356Ser variant locating in the transmembrane domain possibly alters protein stability along with functions rather than location of abnormal MFSD8 proteins in lysosomes. The c. $1102+2 \mathrm{~T}>\mathrm{C}$ variant at the donor site was presumed to change the splicing pattern, and it thus most likely produced abnormal transcripts as well as truncated proteins. Further in-depth studies involving more functions are warranted. The flaw of this study was that the proband refused to perform optical coherence tomography, and the biomarkers such as the hyperreflective spot, the state of the ellipsoid zone, and the choroidal thickness could be considered as predictive factors for further evaluation of visual function and treatment response [24].

Taken together, this study identified novel compound heterozygous variants, c.1066C $>\mathrm{T}$ (p.Pro356Ser) and c. $1102+2 \mathrm{~T}>\mathrm{C}$, in the MFSD 8 gene. They are likely responsible for the isolated macular dystrophy phenotype in this family. Whole exome sequencing is likely to be an effective strategy for screening potentially causative variants of retinopathies with genetic heterogeneity and phenotypic variability. The results further broaden the MFSD 8 gene mutant spectrum and make possible more accurate genetic counseling for this family.

\section{Data Availability}

The data used to support the findings of this study are included in the article.

\section{Conflicts of Interest}

The authors confirm that there are no conflicts of interest.

\section{Acknowledgments}

The authors thank all the subjects and investigators for their contributions to this research. This study was supported by grants from the Natural Science Foundation of Hunan Province (2019JJ50927, 2020JJ3057, and 2020JJ4830), National Natural Science Foundation of China (81873686, 81800219, and 81873780), Special Emergency Project from the Science and Technology Department of Hunan Province (2020SK3032), Distinguished Professor of the Lotus Scholars Award Program of Hunan Province (HD), Education Department Project of Hunan Province (19A058 and 19B072), Emergency Project of Changsha City (kq2001029), Scientific Research Fund for the Talent Introduction of Changsha Medical University, and Application Characteristic Discipline of Hunan Province, China. 


\section{References}

[1] F. Coppieters, G. Ascari, K. Dannhausen et al., "Isolated and syndromic retinal dystrophy caused by biallelic mutations in RCBTB1, a gene implicated in ubiquitination," The American Journal of Human Genetics, vol. 99, no. 2, pp. 470-480, 2016.

[2] X. Liu, T. Tao, L. Zhao, G. Li, and L. Yang, "Molecular diagnosis based on comprehensive genetic testing in 800 Chinese families with non-syndromic inherited retinal dystrophies," Clinical and Experimental Ophthalmology, vol. 49, no. 1, pp. 46-59, 2021.

[3] A. B. Renner and U. Kellner, "Hereditary macular dystrophies," Klinische Monatsblätter für Augenheilkunde, vol. 233, no. 10, pp. 1124-1141, 2016.

[4] L. Wickham, F. K. Chen, G. P. Lewis et al., "Clinicopathological case series of four patients with inherited macular disease," Investigative Opthalmology and Visual Science, vol. 50, no. 8, pp. 3553-3561, 2009.

[5] M. Michaelides, D. M. Hunt, and A. T. Moore, "The genetics of inherited macular dystrophies," Journal of Medical Genetics, vol. 40, no. 9, pp. 641-650, 2003.

[6] A. Hosseini Bereshneh and M. Garshasbi, "Novel in-frame deletion in MFSD8 gene revealed by trio whole exome sequencing in an Iranian affected with neuronal ceroid lipofuscinosis type 7: a case report," Journal of Medical Case Reports, vol. 12, no. 1, Article ID 281, 2018.

[7] P. Steenhuis, J. Froemming, T. Reinheckel, and S. Storch, "Proteolytic cleavage of the disease-related lysosomal membrane glycoprotein CLN7," Biochimica et Biophysica Acta (BBA)_Molecular Basis of Disease, vol. 1822, no. 10, pp. 1617-1628, 2012.

[8] S. Roosing, L. I. van den Born, R. Sangermano et al., "Mutations in MFSD8, encoding a lysosomal membrane protein, are associated with nonsyndromic autosomal recessive macular dystrophy," Ophthalmology, vol. 122, no. 1, pp. 170-179, 2015.

[9] Q. Xiang, Y. Guo, Y. Cao et al., "Identification of a CNGB1 frameshift mutation in a Han Chinese family with retinitis pigmentosa," Optometry and Vision Science, vol. 95, no. 12, pp. 1155-1161, 2018.

[10] X. Huang, L. Yuan, H. Xu et al., "Identification of a novel mutation in the ABCA4 gene in a Chinese family with retinitis pigmentosa using exome sequencing," Bioscience Reports, vol. 38, no. 2, Article ID BSR20171300, 2018.

[11] Q. Xiang, L. Yuan, Y. Cao, H. Xu, Y. Li, and H. Deng, "Identification of a heterozygous mutation in the TGFBI gene in a hui-Chinese family with corneal dystrophy," Journal of Ophthalmology, vol. 2019, Article ID 2824179, 7 pages, 2019.

[12] W. Zheng, H. Chen, X. Deng et al., "Identification of a novel mutation in the titin gene in a Chinese family with limb-girdle muscular dystrophy 2J," Molecular Neurobiology, vol. 53, no. 8, pp. 5097-5102, 2016.

[13] F.-O. Desmet, D. Hamroun, M. Lalande, G. Collod-Béroud, M. Claustres, and C. Béroud, "Human Splicing Finder: an online bioinformatics tool to predict splicing signals," Nucleic Acids Research, vol. 37, no. 9, p. e67, 2009.

[14] G. Yeo and C. B. Burge, "Maximum entropy modeling of short sequence motifs with applications to RNA splicing signals," Journal of Computational Biology: A Journal of Computational Molecular Cell Biology, vol. 11, no. 2-3, pp. 377-394, 2004.

[15] S. Richards, N. Aziz, S. Bale et al., "Standards and guidelines for the interpretation of sequence variants: a joint consensus recommendation of the American College of Medical Genetics and Genomics and the Association for Molecular
Pathology," Genetics in Medicine, vol. 17, no. 5, pp. 405-423, 2015.

[16] Q. Xiang, Y. Cao, H. Xu et al., "Identification of novel pathogenic ABCA4 variants in a Han Chinese family with Stargardt disease," Bioscience Reports, vol. 39, no. 1, Article ID BSR20180872, 2019.

[17] A. Mohammed, M. B. O’Hare, A. Warley, G. Tear, and R. I. Tuxworth, "In vivo localization of the neuronal ceroid lipofuscinosis proteins, CLN3 and CLN7, at endogenous expression levels," Neurobiology of Disease, vol. 103, pp. 123-132, 2017.

[18] T. Danyukova, K. Ariunbat, M. Thelen, N. Brocke-Ahmadinejad, S. E. Mole, and S. Storch, "Loss of CLN7 results in depletion of soluble lysosomal proteins and impaired mTOR reactivation," Human Molecular Genetics, vol. 27, no. 10, pp. 1711-1722, 2018.

[19] W. Jankowiak, L. Brandenstein, S. Dulz, C. Hagel, S. Storch, and U. Bartsch, "Retinal degeneration in mice deficient in the lysosomal membrane protein CLN7," Investigative Opthalmology and Visual Science, vol. 57, no. 11, pp. 4989-4998, 2016.

[20] J. Birtel, M. Gliem, E. Mangold et al., "Next-generation sequencing identifies unexpected genotype-phenotype correlations in patients with retinitis pigmentosa," PLoS One, vol. 13, no. 12, Article ID e0207958, 2018.

[21] K. N. Khan, M. E. El-Asrag, C. A. Ku et al., "Specific alleles of CLN7/MFSD8, a protein that localizes to photoreceptor synaptic terminals, cause a spectrum of nonsyndromic retinal dystrophy," Investigative Opthalmology and Visual Science, vol. 58, no. 7, pp. 2906-2914, 2017.

[22] L. C. Patiño, R. Battu, O. Ortega-Recalde et al., "Exome sequencing is an efficient tool for variant late-infantile neuronal ceroid lipofuscinosis molecular diagnosis," PLoS One, vol. 9, no. 10, Article ID e109576, 2014

[23] E. Siintola, M. Topcu, N. Aula et al., "The novel neuronal ceroid lipofuscinosis gene MFSD8 encodes a putative lysosomal transporter," The American Journal of Human Genetics, vol. 81, no. 1, pp. 136-146, 2007.

[24] I. Ceravolo, G. W. Oliverio, A. Alibrandi et al., "The application of structural retinal biomarkers to evaluate the effect of intravitreal ranibizumab and dexamethasone intravitreal implant on treatment of diabetic macular edema," Diagnostics, vol. 10, no. 6 , p. 413, 2020. 